\title{
Implicaciones de la Ley de Protección de Datos Personales en la gestión preventiva en seguridad y salud en el trabajo
}

\section{Implications of the law of protection of personal data in the preventive management in occupational safety and health}

\author{
Angélica Acevedo Gómez \\ Abogada por la Universidad Santo Tomás Seccional Bucaramanga; especialista en Salud Ocupacional y Riesgos \\ Laborales por la Universidad Manuela Beltrán, Bucaramanga, Colombia; abogada senior, Seguridad y Salud en el \\ Trabajo Pilonietalvarez Abogados \\ Correo electrónico: angelicacevedo17@hotmail.com
}

\begin{abstract}
Resumen
La Ley 1581 de 2012 que dicta disposiciones generales para la protección de datos personales, garantizando tanto el derecho a la intimidad y a la información, de los artículos 15 y 20 de la Constitución, respectivamente, impacta de manera directa en la gestión preventiva que deben realizar todos los encargados de implementar la seguridad y salud en el trabajo en Colombia, por la responsabilidad adicional que cae sobre ellos respecto de los datos personales de la población objetivo.
\end{abstract}

Palabras clave: Protección de datos personales, gestión preventiva, seguridad y salud, trabajo.

\begin{abstract}
Law 1581 of 2012, which provides general provisions for the protection of personal data, guaranteeing both the right to privacy and information in articles 15 and 20 of the Constitution respectively, has a direct impact on the preventive management that must be carried out by all managers To implement Safety and Health at Work in Colombia, for the additional responsibility that falls on them regarding the personal data of the target population.
\end{abstract}

Keywords: Protection of personal data, preventive management, safety and health, work.

\section{Résumé}

Loi 1581 de 2012 qui prévoit des dispositions générales relatives à la protection des données personnelles, assurant à la fois le droit à la vie privée et de l'information de l'article 15 et 20 de la Constitution, respectivement, les impacts directement sur la gestion préventive devrait faire tous les frais pour mettre en œuvre la sécurité et la santé au travail en Colombie, par la responsabilité supplémentaire qui tombe sur eux pour les données personnelles de la population cible.

Mots-clés: Protection des données personnelles, la gestion préventive, la sécurité et la santé, le travail. 



\section{Implicaciones de la Ley de Protección de Datos Personales en la gestión preventiva en seguridad y salud en el trabajo}

Angélica Acevedo Gómez

\section{INTRODUCCIÓN}

Con la expedición del Decreto 1295 de 1994, norma de obligatorio estudio para comprender el Sistema General de Riesgos Laborales, en su artículo 56 establecía cómo el Gobierno Nacional era el responsable de expedir la reglamentación técnica que garantizara la seguridad de los trabajadores en la prevención de accidentes de trabajo y enfermedades profesionales'.

En el 2002, la Organización Internacional del Trabajo publicó las directrices relativas a los Sistemas de Gestión de la Seguridad y Salud en el Trabajo, en el acápite respectivo a la Organización del SG-SST al regular los aspectos propios de la documentación, en el numeral 3.5.4 manifiesta claramente lo siguiente:

Los trabajadores deberían tener el derecho de consultar los registros relativos a su medio ambiente de trabajo y su salud, a reserva de que se respeten los requisitos de confidencialidad (Organización Internacional del Trabajo, 2002, p. 23).

Aunque este documento no tiene un carácter de obligatoria aplicación para los miembros de la OIT resulta claro cómo desde este momento este organismo comprende que la gestión preventiva en seguridad y salud en el trabajo no puede sustraerse de la protección de la información que tiene toda la población objetivo, que serían en principio los trabajadores.

En Colombia solo con la expedición de la Ley 1562 de 2012, 10 años después, se constituye la obligatoriedad de todas las empresas de tener implementado un SGSST en reemplazo del Programa de Salud Ocupacional creado 28 años atrás por el Decreto 614 de 1984, reglamentado por el Ministerio del Trabajo a través de la Resolución 1016 de 1989, no obstante, una de las características que compartían

1 Así se denominaban para la época las enfermedades laborales, término que fue modificado con la expedición de la Ley 1562 de 2012 por "Enfermedades Laborales". 
tanto el PSO como el SG-SST hasta ese momento era el silencio, respecto de la confidencialidad de la información de la población objetivo.

Con la expedición del Decreto 1443 de 2014, respecto de la documentación, el artículo 13 de esta normatividad, manifiesta la obligación del empleador de conservar los registros y documentos que soportan el SG-SST, así mismo, señala que el responsable del SG-SST tendrá acceso a toda la documentación, con excepción de la historia clínica ocupacional cuando no tenga perfil de médico especialista en SG-SST.

De la anterior referencia, puede extractarse cómo el Decreto 1443 de 2014 no tuvo en cuenta las disposiciones de la Ley Estatutaria 1581 de 2012 sobre la obligatoriedad de proteger los datos personales de la población objetivo del SGSST, responsabilidad que adicionalmente debería tener tanto la empresa como el responsable de su desarrollo, máxime, si dentro de la etapa de la planificación debe tenerse en cuenta la descripción socio demográfica de los trabajadores que obliga a recopilar características, como: grado de escolaridad, ingresos, lugar de residencia, composición familiar, estrato socioeconómico, estado civil, raza, ocupación, área de trabajo, edad, sexo y turno de trabajo son datos que al momento de analizar el artículo 5 de la Ley Estatutaria en mención pueden tener la característica de sensibles.

\section{EL EMPLEADOR COMO RESPONSABLE DE LOS DATOS PERSONALES DE SUS TRABAJADORES}

La Superintendencia de Industria y Comercio en consulta contestada bajo el Rad: 13-051560-00001-0000 respondió lo siguiente:

De acuerdo con lo anterior se puede concluir que la Ley 1581 de 2012 resulta aplicable al tratamiento que de los datos personales lleven a cabo personas y entidades tanto de naturaleza pública como privada, incluso, según usted consulta, cuando sean bases de datos a cargo de los empleadores que contengan información de los empleados.

Aunque según el Decreto 1443 de 2014 no solo el empleador tiene obligaciones en materia de SST sino también los contratantes, las organizaciones de economía solidaria y del sector cooperativo junto con las empresas de servicios temporales debe tenerse en cuenta que las obligaciones en materia de seguridad y salud en el trabajo (antes llamada Salud Ocupacional) empezó con las relaciones laborales, tal como se evidencia en las disposiciones de la Ley 57 de 1915; 46 de 1918; 37 de 1921 y 10 de 1934.

Por tal motivo, en este artículo únicamente se hará referencia a la responsabilidad de los empleadores sobre los datos personales de sus trabajadores en lo referido a la gestión preventiva en SG-SST, no sin antes aclarar que las personas naturales al tener la posibilidad de ostentar cualquier calidad contractual (cooperado, contratista, etc.) también tienen el derecho fundamental al habeas data y por ende a la protección de sus datos personales. 


\section{LOS DATOS PERSONALES EN LA LEGISLACIÓN DE SEGURIDAD Y SALUD EN EL TRABAJO}

Los datos personales de los trabajadores han sido abordados en diversas normativas en seguridad y salud en el trabajo como las siguientes:

a. La Resolución 2646 de 2008 dentro de su objeto deja claro que el campo de aplicación al empleador se refiere a la "identificación, evaluación, prevención, intervención y monitoreo permanente de la exposición a los factores de riesgo psicosocial en el trabajo".

En materia de identificación del riesgo psicosocial la Resolución explica cómo deben tenerse en cuenta factores, tanto extra laborales como intra laborales, sin embargo, deja claro en su artículo 10 la utilización de un instrumento particular de evaluación:

Artículo 10. Instrumentos para la evaluación de los factores psicosociales. Los factores psicosociales deben ser evaluados objetiva y subjetivamente, utilizando los instrumentos que para el efecto hayan sido validados en el país.

El único instrumento que se encuentra validado en el país para la evaluación de riesgos psicosociales es la "Batería de instrumentos para la evaluación de factores de riesgo psicosocial” (en adelante la "batería”) (ISBN 978-958-8361-93-2) que se expidió hasta el 18 de febrero de 2011 por el Ministerio de la Protección Social (llamado en ese entonces) tal como consta en la página de internet del fondo de riesgos laborales (http://fondoriesgoslaborales.gov.co/Noticias/Noticia.aspx?IdNoticia=154).

Al observar los cuestionarios que contiene la batería de riesgo psicosocial, podemos constar que:

1) Una ficha de datos generales de 19 preguntas.

2) Un cuestionario de estrés de 31 preguntas.

3) Un cuestionario de factores extra laborales de 31 preguntas.

4) Un cuestionario de factores intra laborales forma A de 123 preguntas, aplicable a trabajadores con cargos de jefatura, profesionales o técnicos.

5) Un cuestionario de factores intra laborales forma B de 97 preguntas, aplicable a trabajadores con cargos auxiliares u operativos.

Al analizar cada uno de los cuestionarios puede observarse cómo los mismos tienden a tocar la esfera de los datos sensibles del trabajador, aspecto que obligaría a obtener una autorización previa y expresa para su tratamiento, todos los datos que no tengan la categoría de públicos deben tener autorización de sus titulares, aspecto que se encuentra claramente dentro de esta encuesta.

Ahora, aunque con posterioridad a la Ley 1581 de 2012 no se ha establecido por parte del Ministerio del Trabajo algún tipo de autorización especial para la aplicación de la batería de riesgo psicosocial sí es en principio obligación del empleador el poner de presente al trabajador la posibilidad de autorizar el tratamiento de la información. 
Es importante explicar que es posible que se entienda el cumplimiento de la obligación de obtener la autorización con la aplicación del documento técnico completo del Ministerio, al momento de referirse a la obligación de obtener una firma del consentimiento informado que debe dar la persona para participar en el estudio, sin embargo, el mismo no cumple con los requisitos que señala la Ley Estatutaria que debe contener la autorización de la recepción de los datos como el que sea:

1) Previa.

2) Informada.

3) Obtenida por un medio que pueda garantizar su consulta posterior.

De otra parte, es importante tener en cuenta que este consentimiento informado según la Resolución 8430 de 1993, art. 14 es más una autorización para participar en una investigación que un permiso para que el psicólogo le dé tratamiento a sus datos personales, por este motivo, resulta claro cómo el mismo -en materia del tratamiento de datos- no es suficiente para aplicar la batería de riesgo psicosocial.

Esta circunstancia obliga a los expertos, según esta resolución, que son los psicólogos con licencia en Salud Ocupacional, a obtener por parte de los trabajadores a quienes se les apliquen estas en cuentas estas autorizaciones, con el fin de evitar la vulneración de su derecho a la intimidad.

b. La Resolución 2346 de 2007 que regula el tema de las historias clínicas ocupacionales.

Esta Resolución es de cierta forma la guía que deben tener los médicos y las IPS con licencia en Salud Ocupacional que se encargan de realizar los exámenes preocupacionales de ingreso, periódicos y de egreso mediante la contratación con los empleadores que tienen tal obligación.

En el artículo 17 de esa Resolución que fue modificado por el artículo 2 de la Resolución 1918 de 1995 deja claro que la custodia de las historias clínicas ocupacionales $^{2}$ es responsabilidad del médico con licencia en Salud Ocupacional que las practica, o de las IPS con licencia en Salud Ocupacional dejando como parámetro regulador de su manejo la norma que regula las historias clínicas en general.

De acuerdo con el artículo 5 de la Ley Estatutaria, los datos relativos a la salud son considerados datos sensibles, de acuerdo con esto, estos profesionales de la salud y las respectivas entidades antes de efectuar la práctica de tales exámenes médicos se encuentran obligados a solicitar por parte de los trabajadores la respectiva autorización para poder realizar sus funciones sin que ello repercuta en la violación de su derecho fundamental a la intimidad.

2 Definida por el artículo 14 de esa resolución como:

"el conjunto único de documentos privados, obligatorios y sometidos a reserva, en donde se registran cronológicamente las condiciones de salud de una persona, los actos médicos y los demás procedimientos ejecutados por el equipo de salud que interviene en su atención. Puede surgir como resultado de una o más evaluaciones médicas ocupacionales. Contiene y relaciona los antecedentes laborales y de exposición a factores de riesgo que ha presentado la persona en su vida laboral, así como resultados de mediciones ambientales y eventos de origen profesional". 
Sobre este punto es importante tener en cuenta lo dispuesto por la Ley $1751 \mathrm{de}$ 2015, que regula el derecho fundamental a la salud:

Artículo 10. Derechos y deberes de las personas, relacionados con la prestación del servicio de salud. Las personas tienen los siguientes derechos relacionados con la prestación del servicio de salud:

k) A la intimidad. Se garantiza la confidencialidad de toda la información que sea suministrada en el ámbito del acceso a los servicios de salud y de las condiciones de salud y enfermedad de la persona, sin perjuicio de la posibilidad, de acceso a la misma por los familiares en los eventos autorizados por la ley o las autoridades en las condiciones que esta determine.

Como puede observarse, los profesionales de la salud, tienen la obligación de guardar la confidencialidad de toda la información que recopilen en el desarrollo de su trabajo -incluyéndose en este caso a los médicos que practican las evaluaciones antes mencionadas- no obstante, esto no los exime y a las IPS que llevan la guarda y custodia de las historias clínicas ocupacionales de suscribir la respectiva autorización para el tratamiento de los datos personales de estas personas, en los términos de la Ley Estatutaria comentada, valga aclarar, que existe una excepción para el requerimiento de la autorización en los términos del artículo 10, literal c de la Ley 1581 de 2012 como es en los casos de urgencia médica o sanitaria, sin embargo, este es un aspecto que no se aplica a la disposición comentada, toda vez que el elemento urgencia no se configura en la práctica de tales evaluaciones médicas.

c. Las resoluciones 2844 de 2007 y 1013 de 2008

A través de estas resoluciones el entonces Ministerio de la Protección Social (hoy Ministerio del Trabajo) adoptó las Guías de Atención Integral de Salud Ocupacional, basadas en la evidencia, a través de la primera las Guías de Dolor lumbar Inespecífico y enfermedad discal relacionados con la manipulación manual de cargas y otros factores de riesgo en el lugar de trabajo; desórdenes músculoesqueléticos relacionados con movimientos repetitivos de miembros superiores (Síndrome de Túnel Carpiano, Epicondilitis y Enfermedad de De Quervain); Hombro doloroso relacionado con factores de riesgo en el trabajo; Neumoconiosis (silicosis, neumoconiosis del minero de carbón y asbestosis) e Hipoacusia neurosensorial inducida por ruido en el lugar de trabajo y a través de la segunda, las guías para Asma ocupacional; trabajadores expuestos a benceno y sus derivados; cáncer pulmonar relacionado con el trabajo; dermatitis de contacto relacionada con el trabajo y trabajadores expuestos a plaguicidas inhibidores de la colinesterasa.

El parágrafo del artículo primero de estas resoluciones es uniforme en decir:

Parágrafo. Las guías de Atención Integral de Salud Ocupacional que se adoptan mediante la presente resolución serán de obligatoria referencia por parte de las entidades promotoras de salud del régimen contributivo y subsidiado, administradoras 
de riesgos profesionales, instituciones prestadoras de servicios de salud, instituciones prestadoras de servicios de salud ocupacional y empleadores, en la prevención de los daños a la salud por causa o con ocasión del trabajo, la vigilancia de la salud, el diagnóstico, tratamiento y rehabilitación de los trabajadores en riesgo de sufrir o que padecen las mencionadas patologías ocupacionales.

De acuerdo con lo anterior, los empleadores deben tener estas guías como un documento de obligatoria consulta al momento de prevenir los riesgos que se encuentren en el trabajo, siempre y cuando estos puedan devenir en alguna enfermedad que las mismas tengan, por ejemplo, en el Decreto 1477 de 2014 que enlista las enfermedades laborales es una herramienta para los empleadores en la prevención de estas, porque permite a través de los agentes de riesgo y las actividades que realizan los trabajadores establecer un origen laboral de la enfermedad.

Por citar un ejemplo, en el caso particular de los "Trabajadores de la industria textil (costureros, empacadores, tejedores y bordadores). Trabajadores de movimientos cultivos de flores" según el Decreto, el agente de riesgo consiste en una "Combinación de movimientos repetitivos con posturas forzadas de miembros superiores, con alta demanda de tareas manuales o con herramientas de vibración", dentro de las enfermedades que podrían diagnosticarse por esa exposición se encuentra el síndrome del túnel del carpo, enfermedad que se aborda en la Guía de atención integral basada en la evidencia para desórdenes musculoesqueléticos (dme) relacionados con movimientos repetitivos de miembros superiores (Síndrome de Túnel Carpiano, Epicondilitis y Enfermedad de De Quervain (GATI- DME)”.

En particular la guía de los DME cuando se empiezan a dar las recomendaciones que permiten identificar si en el puesto de trabajo se encuentra ese factor de riesgo ocupacional plantea que previamente se tengan como estrategias la realización de "encuestas de morbilidad sentida".

Las encuestas de morbilidad sentida son herramientas epidemiológicas que permiten obtener información de la morbilidad no diagnosticada o percibida por parte de la población (González, 2007).

Es decir, estas encuestas permiten identificar porqué pueden enfermarse las personas y qué condiciones individuales podrían aumentar las posibilidades de sufrir una que otra patología. Nuevamente con estas Gatiso surge la necesidad de entregar a los empleadores o a las personas a quienes ellos deleguen para la gestión en seguridad y salud en el trabajo los datos referidos a la salud que están catalogados como sensibles y por ende susceptibles de una autorización como ya se explicó con anterioridad.

d. Decreto 1443 de 2014 (Compilado por el Decreto 1072 de 2015, único reglamentario del sector trabajo).

Esta normatividad que define la organización del SG-SST en todas las empresas. Dentro de la etapa de planeación del sistema, más específicamente al momento de realizar la evaluación inicial, se hace necesario identificar a la población a la cual 
se aplicará, con el fin de definir las estrategias para utilizar, por este motivo se hace imprescindible conocer las descripciones y características sociales y demográficas de los trabajadores, como: grado de escolaridad, ingresos, lugar de residencia, composición familiar, estrato socioeconómico, estado civil, raza, ocupación, área de trabajo, edad, sexo y turno de trabajo, esto según el art. 2, numeral 13 y las condiciones de salud.

De nuevo, en este punto se observa la referencia a datos personales, específicamente en lo concerniente al perfil socio demográfico al hacer referencia a la "raza"3 que genera por parte del encargado del SST, la obligación de poner de presente en la encuesta donde se identifique este perfil, la autorización previa de este dato personal sensible, poniendo de presente que no está obligado a suministrarlo.

Por este motivo, al momento de empezar a implementar el SG-SST no puede sustraerse la necesidad de dar cumplimiento a la Ley 1581 de 2012, estableciendo la respectiva política de protección de datos personales y articular las autorizaciones del tratamiento de estos con los documentos en los cuales se recopile información de los trabajadores.

e. Resolución 1565 de 2014, por la cual se expide la Guía metodológica para la elaboración del Plan Estratégico de Seguridad Vial.

De acuerdo con el artículo 12 de la Ley 1503 de 2011, se ordenó a:

Toda entidad, organización o empresa del sector público o privado que por cumplir sus fines misionales o en el desarrollo de sus actividades posea, fabrique, ensamble, comercialice, contrate o administre flotas de vehículos automotores o no automotores superiores o diez (10) unidades, o contrate o administre personal de conductores o diseñar un Plan Estratégico de Seguridad Vial.

Esta obligación se genera porque Colombia se unió al "Decenio de acción para la seguridad vial 2011-2020" de la OMS, con el fin de crear un compromiso de disminuir la ocurrencia de los accidentes de tránsito, podemos observar a través del estudio de esta norma, que el Estado al parecer resulta ser incapaz de controlar a los actores de la vía y a través de la Ley 1503 delega a las empresas relacionadas en la norma anterior, la implementación de un plan estratégico en el que se controle el mantenimiento de los vehículos y la conducta de los conductores.

De acuerdo con lo anterior, dentro de este plan estratégico, se hace necesario llevar un control de la documentación de los conductores que le permita tener un control y registro del cumplimiento de fechas sobre la documentación de ley requerida y la trazabilidad de las acciones dentro del PESV. La guía metodológica, frente a este punto dice de manera literal: "La información y su manejo, debe ajustarse según lo definido en la Ley de Habeas Data”. Esta circunstancia nos muestra cómo de igual

3 De acuerdo con el art. 5 de la Ley 1581 de 2012, el origen racial o étnico es considerado un dato sensible. 
forma el empleador o contratante debe articular su política de protección de datos con los conductores que serán la población objeto del PESV, además de ello, la necesidad de solicitarles previamente a cualquier iniciación de las actividades concernientes al mismo, la autorización para el manejo y tratamiento de sus datos personales.

\section{CONCLUSIONES}

Resulta claro cómo todas las actividades tendientes para realizar la gestión preventiva en seguridad y salud en el trabajo no pueden sustraerse del correcto tratamiento de los datos personales de la población objeto, por este motivo, se hace necesario que las políticas de tratamiento de datos personales incluyan este componente dentro de su contenido y, además de ello, que antes de realizar cualquier actividad tendiente a cumplir las normas de SST se suscriba la respectiva autorización con los trabajadores para efectos de evitar problemas ante la Superintendencia de Industria y Comercio en el futuro.

\section{REFERENCIAS}

Decreto 1072 del 26 de mayo de 2015, "por medio del cual se expide el Decreto Único Reglamentario del Sector Trabajo”, D.O. 49.523.

Decreto 1295 del 22 de junio de 1994, "por el cual se determina la organización y administración del Sistema General de Riesgos Profesionales”, D.O. 41.405

Decreto 614 del 14 de marzo de 1984, "por el cual se determinan las bases para la organización y administración de Salud Ocupacional en el país”, D.O. 36561.

González, G., Valencia, M.L., Agudelo, N.A., Acevedo, L., Vallejo, I.C. (2207). Morbilidad sentida de las urgencias médicas y la utilización de los servicios de salud en Medellín, Colombia, 2005-2006. Recuperado el 12 de diciembre de 2015 de http://www.revistabiomedica.org/index.php/biomedica/article/ viewFile/214/201

Ley 1562 del 11 de julio de 2012, "por la cual se modifica el Sistema de Riesgos Laborales y se dictan otras disposiciones en materia de Salud Ocupacional", D.O. 48.488

Ley 1581 del 17 de octubre 2012 "por la cual se dictan disposiciones generales para la protección de datos personales", D.O. 48.587.

Organización Internacional del Trabajo. (2002). Directrices relativas a los Sistemas de Gestión de la Seguridad y Salud en el Trabajo, ILO-OSH 2002, recuperado el 12 de diciembre de $2015 \mathrm{de}$ http://www.ilo.org/wcmsp5/groups/public/---ed_protect/--protrav/---safework/documents/normativeinstrument/wcms_112582.pdf-

Organización Mundial de la Salud. (s.f.). Decenio de acción para la seguridad vial 2011-2020. Recuperado de https://www.google.com.co/url?sa=t\&rct=j\&q= $\&$ esrc $=$ s\&source $=$ web\& $c d=1 \& \operatorname{cad}=$ rja\&uact $=8 \& v e d=0$ ahUKEwiMzZfxz 
dbJAhXDeSYKHZa-Du0QFggbMAA\&url=http\%3A\%2F\%2Fwww.who. int $\% 2$ Froadsafety $\% 2$ Fdecade_of_action $\% 2 \mathrm{Fes} \% 2 \mathrm{~F} \&$ usg=AFQjCNH5ADV ABIuEBcJC1XeKy3sNy-S0eQ\&sig2=4jk6Ukpun-7MD5OIMV_kFA 12 de diciembre de 2015.

Resolución 1013 del 25 de marzo de 2008, "por la cual se adoptan las Guías de Atención Integral de Salud Ocupacional Basadas en la Evidencia para Asma Ocupacional, trabajadores expuestos a benceno, plaguicidas inhibidores de la colinesterasa, dermatitis de contacto y cáncer pulmonar relacionados con el trabajo", D.O. 46.943

Resolución 1016 del 31 de marzo de 1989, "por la cual se reglamenta la organización, funcionamiento y forma de los Programas de Salud Ocupacional que deben desarrollar los patronos o empleadores en el país",

Resolución 1564 del 6 de junio de 2014, "por la cual se expide la Guía metodológica para la elaboración del Plan Estratégico de Seguridad Vial”, D.O. 49.177

Resolución 2346 del 11 de julio de 2007, "por la cual se regula la práctica de evaluaciones médicas ocupacionales y el manejo y contenido de las historias clínicas ocupacionales", D.O. 46691

Resolución 2646 del 16 de julio de 2008, "por la cual se establecen disposiciones y se definen responsabilidades para la identificación, evaluación, prevención, intervención y monitoreo permanente de la exposición a factores de riesgo psicosocial en el trabajo y para la determinación del origen de las patologías causadas por el estrés ocupacional", D.O. 47059.

Resolución 2844 del 16 de agosto de 2007, "por la cual se adoptan las Guías de Atención Integral de Salud Ocupacional Basadas en la Evidencia", D.O. 46.728

Superintendencia de Industria y Comercio. (2013). Concepto 13-037288 del 5 de abril de 2013. Recuperado el 12 de diciembre de 2015 de https://www.google. com.co/url?sa $=$ t\&rct $=\mathrm{j} \& \mathrm{q}=\&$ esrc $=\mathrm{s} \&$ source $=$ web $\& \mathrm{~cd}=1 \& \mathrm{cad}=$ rja \&uact $=8 \&$ ved=0ahUKEwi--MGQzNbJAhUBcSYKHWVyBasQFggcMAA\&url=http\%3A\%2F\%2Fwww.sic.gov.co\%2Fdrupal\%2Fsites\%2Fdefault $\% 2 \mathrm{Ffiles} \% 2 \mathrm{~F}-$ normatividad\%2FConcepto_13-37288.pdf\&usg=AFQjCNGftDswPcSZBKrYaW0z3mua2SSyNA\&sig2=tRfudKttB-1Z-uK9RSXuqg 